\title{
Development of a Standard Methodology for Documenting Processes in an Organization
}

\section{Desarrollo de una Metodología Estándar para el Levantamiento de Procesos en una Organi-} zación

\author{
G. Alarcon, P. Alarcon, M. Altamirano, and E. Centeno
}

Escuela Superior Politécnica de Chimborazo, Riobamba, Ecuador

VII International Congress of

Science, Technology,

Entrepreneurship and

Innovation (SECTEI 2020)

Corresponding Author:

G. Alarcon

ingiovannyalarcon@hotmail.com

Published: 26 August 2021

Production and Hosting by

Knowledge E

(c) G. Alarcon et al. This article is distributed under the terms of the Creative Commons Attribution License, which permits unrestricted use and redistribution provided that the original author and source are credited.
S OPEN ACCESS

\section{Abstract}

The application of process management in our country has been extended throughout the last decade; improvement in management, high performance levels, reduction of operation times, optimization of resources and obtaining of quality certifications are among the main reasons why both public and private organizations seek to implement it. However, despite being an implicit activity within the change of management model to the process approach and even the improvement of the functional administrative model, the documentation of processes becomes an uncomfortable, complex and time-consuming task that often generates nervousness and distrust in workers. Diversity of criteria and the lack of an adequate methodology with effective tools makes it difficult to achieve the expected results. This article presents in detail the steps, their sequence and instruments used that allow an organization to raise the information of its processes and document them. As a result of different research projects developed over several years, the methodology of process mapping allowed for the documentation of all the activities of different administrative units of the Escuela Superior Politécnica de Chimborazo and not only to comply with the laws and regulations but also to improve their management. In the last application, it contributed to the development of the documentary structure of a quality management system that allowed an academic unit to achieve international quality certification.

Keywords: management, processes, process documentation, process manual.

Resumen

La aplicación de la gestión de procesos en nuestro país se ha ido extendiendo a lo largo de la última década; mejoras en la gestión, altos niveles de rendimiento, reducción de tiempos de operaciones, optimización de recursos y obtenciones de certificaciones de calidad son entre otros los principales motivos por los cuales organizaciones tanto públicas como privadas buscan implementarla. Sin embargo y a pesar de ser una actividad implícita dentro del cambio de modelo de gestión hacia el enfoque de procesos e incluso del mejoramiento del modelo administrativo funcional, la documentación de los procesos se convierte en una labor incomoda, compleja y con un alto consumo de tiempo que suele generar nerviosismo y desconfianza en los trabajadores. Diversidad de criterios y la falta de una metodología adecuada con instrumentos eficaces complica cumplir los resultados esperados. El presente artículo presenta a detalle los pasos, su secuencia e instrumentos utilizados que le permiten a una organización levantar la información de sus procesos y documentarlos. Resultado de diferentes proyectos de investigación desarrollados a los largo de varios años, la metodología de levantamiento de procesos permitió documentar todas las actividades de distintas unidades administrativas de la Escuela Superior Politécnica de Chimborazo y no solo cumplir lo 
establecido el leyes y reglamentos sino también mejorar su gestión, en la última aplicación contribuyó a elaborar la estructura documental de un sistema de gestión de la calidad que logró que una unidad académica alcance una certificación internacional de calidad.

Palabras Clave: gestión, procesos, documentación de procesos, manual de procesos.

\section{Introducción}

\subsection{La documentación en las organizaciones}

Desde la formalización de las operaciones de las empresas a finales de la revolución industrial cuando investigadores y estudiosos de la administración generaban teorías administrativas dando inicio a la necesidad de registrar los principios administrativos que regían las actividades empresariales para contar con una evidencia documental, hasta nuestros días en que nuevas propuestas administrativas se implementan cada día; la documentación ha ido adquiriendo cada vez mayor importancia debido a que no solamente refleja la estructura, actividades, niveles de responsabilidad [1] de la empresa entre otros, sino que son empleados también para actividades de control, revisión y mejora al interior de la misma [2], además de organismos externos con distintos fines.

Uno de los considerados padres de la administración, el ingeniero francés Henri Fayol (1841-1925) publicó en el año 1917 su obra 'Administración Industrial y General'; este libro presentaba el resultado de sus estudios realizados sobre el funcionamiento de la empresa a partir de las experiencias adquiridas como gerente de la Compagnie Commentry Fourchambault et Decazeville. Su aporte permitió a los administradores de la época e incluso actuales orientar las actividades de dirección fundamentados en los principios administrativos descritos en su obra para alcanzar los objetivos empresariales propuestos [3]; principios administrativos como la unidad de mando, autoridad y responsabilidad, unidad de dirección, centralización, disciplina, división del trabajo, orden, jerarquía [4] entre otros, que debían estar debidamente documentados y comunicados para que todos en la empresa los conozcan y puedan aplicarlos.

Resultado de esta práctica se inició una cultura de documentación y generación de evidencias de las actividades empresariales, ahora denominada como gestión de la información [5] que además se considera en nuestros días como patrimonio documental de la empresa [6]; en el trabajo denominado Gestión del conocimiento y gestión de la información, los autores identifican tres tipos de documentos:

- Interna: Hace referencia a aquella documentación generada o recibida por la organización en el ejercicio de sus funciones, es decir, son documentos que 
surgen de la actividad diaria de esa institución. En este grupo, encontramos tanto la documentación típicamente administrativa: Contabilidad, correspondencia,... , como la documentación de gestión: Informes, actas de reuniones, procedimientos de trabajo, y la documentación técnica que refleja la propia actividad de la organización.

- Externa: Además de la documentación producida por la propia organización, ésta y las personas que trabajan en ella necesitan, a menudo, consultar y manejar fuentes de información externas: Libros revistas, B.D., Internet.

- Pública: Es aquella documentación que la organización produce de cara al público, para comunicarles hechos, actividades, acontecimientos,... por ejemplo, las memorias, los catálogos de productos y servicios, la página web,... [3].

La compilación de la información interna es la que da origen a los manuales de gestión o instrumentos de gestión, documentos establecidos por la alta dirección de la empresa [7] cuyo propósito no solo es documentar las actividades sino guiarlas, logrando convertiste en fuentes de información para los trabajadores y fundamento para actividades de capacitaciones e inducción a nuevas incorporaciones. Además de lo expuesto en líneas anteriores son usados para control ya sea interno o externo. Existe diferentes tipos de documentación interna que emplea una empresa, desde manuales de organización y funciones a planificaciones estratégicas, manuales de sistemas y procedimientos, presupuestos operativos y financieros, instructivos entre otros forman parte del quehacer empresarial.

De pendiendo de la naturaleza, cultura organizacional y modelo [8] de gestión, cada organización usa uno o varios tipos de documentación, siendo el mas usado el manual de gestión de la organización.

En el caso de la organizaciones que aplican la administración funcional el manual de gestión toma el nombre de manual de funciones, este modo administrativo se caracteriza por un establecimiento de responsabilidades por funciones [9] basado en el principio de jerarquía, de tal forma que quién está en la cima de la empresa es el que ejerce la unidad de mando, planificando, organizando y distribuyendo el trabajo a todos los funcionarios, Chica, Villada y Restrepo definen al manual de funciones como:

[...] un instrumento de administración de personal a través del cual se establecen las funciones, las competencias laborales de los empleos que conforman la planta de personal de una entidad y los requerimientos exigidos para el desempeño de estos. Es una herramienta que da soporte técnico para justificar y dar sentido a la existencia de los cargos en una entidad $u$ organismo. [10]

La alternativa de modelo de gestión a la administración funcional es la gestión por procesos, cuyos principios se basan en la autogestión, enfoque hacia la satisfacción 
de los clientes, trabajo en equipo y colaborativo en la búsqueda de mejores resultados [11]. Para las organizaciones que emplean la gestión por procesos, el manual de gestión organizacional toma el nombre de manual de procesos (llamado también manual de procedimientos), en él se documentan detalladamente toda la información de los procesos que cada colaborador desarrolla en la empresa [12], los objetivos, necesidades de recursos, procedimientos entre otros, se referencia a Muñoz Álvarez quién establece su importancia indicando que:

El manual de procesos y procedimientos tiene como propósito fundamental servir de soporte para el desarrollo de las acciones, que en forma cotidiana la entidad debe realizar, a fin de cumplir con cada competencia particular asignadas por mando constitucional o legal, con la misión fijada y lograr la visión trazada. [13]

En los dos tipos de administración o gestión, el levantamiento de procesos es la actividad por la cual se logra generar los documentos de gestión descritos.

\subsection{La documentación y la gestión de la calidad}

Es amplia la literatura que describe el uso e importancia de la documentación de procesos, sin embargo es ha raíz del aparecimiento y difusión de las normas de calidad cuando su aplicación va mas allá de tener documentadas las actividades y se transforman en verdaderos instrumentos de gestión y mejora en la empresa. Tal resulta ser su importancia que las auditorías de conformidad en los sistema de gestión se fundamentan en la revisión de los la información documentada, la cual puede estar en cualquier tipo de medio o soporte [14], es decir de manera física o digital, pero que logre demostrar la planificación y ejecución de las actividades de la empresa conforme la planificación y en cumplimiento de los requerimientos [15] de las partes interesadas o clientes.

En el caso de las actividades de control interno o denominadas también de primera parte, la documentación es respaldo [16] de las acciones y decisiones tomadas; fundamental para la gestión eficiente, este tipo de auditoría es el primer paso para la demostración de conformidad documental que una organización evidencia en el cumplimento de determinado estándar, en el trabajo denominado 'Auditorías, Mejora Continua y Normas ISO: Factores clave para la evolución de las organizaciones' sus autores se refieren a ella de la siguiente manera:

Por su parte ${ }^{* *}$ Gonzalbes y Medina (2003), enfocan los tipos de auditoria según los elementos que intervienen en la misma, siendo este uno de los más utilizados actualmente e inclusive por la norma ISO 19011: 2011, Directrices para la auditoria de los sistemas de gestión, donde se considera a la auditoría interna, denominada también 
por muchos autores como auto- auditorias o auditoria de primera parte. Por su parte, ${ }^{* *}$ Cuatrecasa (2010), la define como aquellas que se elaboran en la propia empresa, a solicitud de la alta dirección. Se llevara a cabo con personal cualificado que actuará como auditor con el objeto de realizar una autoevaluación de la propia empresa [17] (p. 347).

Este 'auto control', realizado de manera planificada y periódica podrá dotar a la organización de la evidencia necesaria para no solo demostrar conformidad con algún estándar en particular sino sobre todo mejorar los procesos [18] en base a mediciones objetivas [19]. En una aplicación de este tipo de auditoría, resalta aquella que audita los procesos, su cumplimiento y eficacia, citado por Yánez y Yánez (2012), **Parsowith (1999) en su trabajo señala que:

[...] resaltando la importancia de las auditorias de procesos, conocida también como auditoria de controles de procesos, de operaciones o de una serie de operaciones; donde se evalúa los procedimientos establecidos, a través de la verificación de su existencia o instrucciones de trabajo para los procesos y/o actividades que se realizan en la organización. [17]

Por lo expresado, es fundamental que los procesos estén debidamente documentados y actualizados como parte de la planificación y actividades de control para la mejora continua.

\subsection{La documentación de los procesos}

En el trabajo denominado 'La gestión por procesos: Su papel e importancia' el autor escribe sobre la nueva tendencia que a nivel mundial se instituía hacia el inicio de los años 2000; expresaba que:

[...] han surgido varios modelos de gestión basados en los procesos. Por su aceptación extendida, por los resultados que están dando en bastantes organizaciones y por su integración creciente con otros modelos de gestión como el marketing relacional y el modelo europeo (EFQM) de excelencia empresarial. [20]

En el trascurso de este tiempo son abundantes las experiencias que testifican como el modelo de gestión por procesos ha permitido una mejora evidente en la gestión de la organización y obtención de mejores resultados [21], y como todo modelo de gestión debe articular un conjunto de documentos que ordenados y jerarquizados de manera adecuada reflejen la estructura y funcionamiento de la empresa. Fernández (1986) describió lo que a su juicio es una estructura empresarial: 
La estructura representa un sistema estable de relaciones entre los miembros de una organización. Constituye, por tanto en marco donde se desarrollan los procesos interno de esta, en especial de la toma de decisiones, lo que la convierte en un elemento determinante del comportamiento organizativo.

Es por lo tanto la documentación de las actividades (procesos internos) un componente fundamental de la estructura organizativa, en donde 'los procedimientos, las tareas y las responsabilidades están claramente descritas y definidas en documentos o manuales de operación, siendo predecible la dinámica de las diferentes unidades organizativas' [23], por lo tanto en la gestión de procesos, los procedimientos documentados forman parte de la estructura documental [24] que debe tener una organización para evidenciar de su modelo de gestión aplicado pero fundamentalmente como una estrategia para la mejora continua.

Por lo expuesto, contar con una metodología adecuada e instrumentos eficaces que permitan recolectar apropiadamente la información del desarrollo de las actividades que cada uno de los colaboradores de la organización realiza se convierte en estratégico, pues la empresa no solo dispondrá de la información documentada para los fines antes descritos, sino que podrá iniciar un camino hacia la mejora continua y el cumplimiento de estándares de calidad, pues aquellas empresa certificadas bajo normas de calidad tienen sus procesos de trabajo más formalizados, resultado lógico teniendo en cuenta que la certificación lleva a las empresas a documentar sus procesos [25].

Con estos antecedentes, el presente artículo tiene por objetivo proponer una metodología estándar tanto de procedimiento como instrumentos de recolección de información que le permita a un organización realizar el levantamiento de procesos con fines de documentación e incluso como apoyo en la implementación de la gestión de procesos en una organización.

\section{Metodología}

El levantamiento de procesos es la actividad por la cual se documentan las actividades dentro de cada uno de los procesos que desarrolla un colaborador (trabajador) en una empresa, con el nivel de detalle necesario para que pueda ser reproducido por un trabajador; metodológicamente es una entrevista por la cual se logra identificar todas aquellas actividades que se ejecutan, incluso aquellas que no se encuentran en reglamentos, estatutos y manuales de funciones. En el trabajo denominado 'La entrevista, recurso flexible y dinámico', sus autores la definen como 'una conversación que se propone con un fin determinado distinto al simple hecho de conversar. Es un instrumento técnico de gran utilidad en la investigación cualitativa, para recabar 
datos' [26]. De los tres tipos de entrevista mas usadas, las entrevistas estructuradas o enfocadas, entrevistas semiestructuradas y entrevistas no estructuradas; de estas el segundo tipo es la más recomendada para el levantamiento de la información de los procesos debido a su capacidad de apartarse a las condiciones que se presentan en la que el entrevistador no puede anticipar las respuesta y necesariamente debe darle libertad al entrevistado (trabajador) para interactuar; al respecto:

Entrevistas semiestructuradas: presentan un grado mayor de flexibilidad que las estructuradas, debido a que parten de preguntas planeadas, que pueden ajustarse a los entrevistados. Su ventaja es la posibilidad de adaptarse a los sujetos con enormes posibilidades para motivar al interlocutor, aclarar términos, identificar ambigüedades y reducir formalismos. [26]

La propuesta metodológica requiere la consecución de tres etapas claramente definidas:

- Planificación del Levantamiento de Procesos;

- Levantamiento de procesos (Etapa principal);

- Documentación de los procesos.

\subsection{Primera etapa: Planificación del levantamiento de procesos}

En esta primera parte se debe planificar la actividad del levantamiento de procesos a partir del dimensionamiento del trabajo, considerando el análisis de los documentos de la empresa que permita elaborar la planificación, cronograma de visitas y aprobación. Como se ha mencionado a lo largo del documento la documentación de procesos es una estrategia de calidad, y la calidad requiere planificación.

\subsubsection{Análisis documental de la estructura organizacional}

El primer paso previo al levantamiento de información es la revisión de los documentos que contengan información respecto de la organización de la empresa y la distribución de responsabilidades; esta actividad permite identificar el número de personas que forman parte de la organización y los puestos de trabajo. El estatuto, normativas de constitución, manual de funciones e incluso el organigrama son entre otros los principales los documentos que proveen la información preliminar. Con la información recabada se debe realizar la matriz de puestos de trabajo, la tabla que se presenta a continuación muestra el detalle de la información extraída.

También y gracias a este análisis documental se identifican los procesos a caracterizar [27] por puesto de trabajo conforme la información contenida en los documentos 
Table 1

Matriz de puesto de trabajo (Aplicación realizada en la Dirección de Desarrollo Academico).

\begin{tabular}{|c|c|c|c|c|c|}
\hline $\mathrm{N}^{\circ}$ & $\begin{array}{l}\text { Denominación del } \\
\text { puesto de trabajo }\end{array}$ & $\begin{array}{l}\text { Colaboradores } \\
\text { por puesto de } \\
\text { trabajo }\end{array}$ & $\begin{array}{l}\text { Área de } \\
\text { departamento }\end{array}$ & gestión & Información contenida en \\
\hline 1 & Director/a & 1 & $\begin{array}{l}\text { Dirección de } \\
\text { Académico }\end{array}$ & Desarrollo & Estatuto Politécnico 2019 \\
\hline 2 & Analista Académica & 1 & $\begin{array}{l}\text { Dirección de } \\
\text { Académico }\end{array}$ & Desarrollo & Descripción \\
\hline 3 & $\begin{array}{l}\text { Analista de Soporte } \\
\text { y Mantenimiento }\end{array}$ & 1 & $\begin{array}{l}\text { Dirección de } \\
\text { Académico }\end{array}$ & Desarrollo & $\begin{array}{l}\text { Clasificación de puestos de trabajo } \\
\text { de la Escuela Superior Politécnica }\end{array}$ \\
\hline 4 & Técnico Docente & 2 & $\begin{array}{l}\text { Dirección de } \\
\text { Académico }\end{array}$ & Desarrollo & $\begin{array}{l}\text { de Chimborazo } 2014 \text { (Resolución. } \\
\text { Administrativa. No.138-ESPOCH- }\end{array}$ \\
\hline 5 & Secretaria & 1 & $\begin{array}{l}\text { Dirección de } \\
\text { Académico }\end{array}$ & Desarrollo & $2016,23 / 09 / 2016)$ \\
\hline
\end{tabular}

analizados y que son de cumplimiento legal y reglamentario, la tabla que se presenta a continuación se constituye en la guía para el levantamiento de procesos a realizar a cada colaborador.

Table 2

Matriz de procesos de trabajo (Aplicación realizada en la Dirección de Desarrollo Academico).

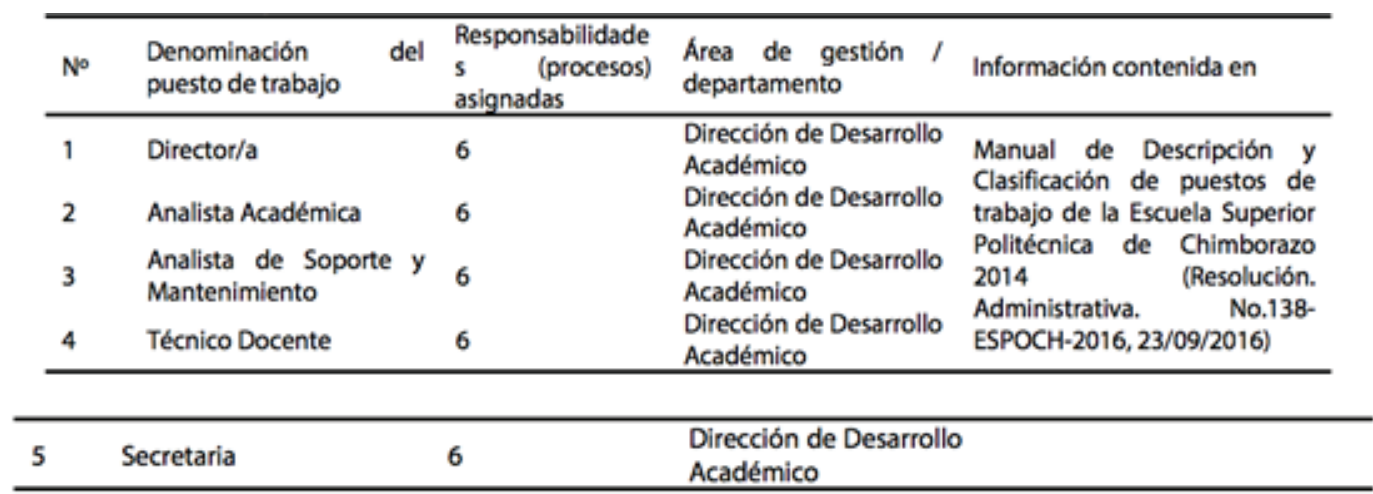

Uno de los aspectos importantes en este punto es determinar el número de trabajadores que existe por puesto de trabajo, en el caso de ser mas de dos trabajadores queda a criterio del planificador del levantamiento de procesos decidir aplicar a todos o extraer una muestra, depende fundamentalmente del número de individuos; sin embargo y considerando que al pertenecer a la misma denominación y que realizan las mismas actividades no es necesario aplicar el instrumento de recolección de datos a todos, una selección por muestro aleatorio simple extrayendo los individuos al azar $[28,29]$ será lo adecuado. A manera de recomendación, la selección de los trabajadores más antiguos en el puesto permitirá recolectar información más confiable y detallada por su mayor conocimiento de las actividades del puesto de trabajo. 


\subsubsection{Elaboración del Plan de levantamiento de procesos}

Una vez determinado los trabajadores que serán objeto del levantamiento de información se debe elaborar un Plan que debe incluir los siguientes elementos:

- Objetivo de la actividad

- Alcance

- Equipo responsable

- Trabajadores seleccionados

- Cronograma de entrevista

\subsubsection{Aprobación del levantamiento de procesos}

Debido a que el levantamiento de procesos es fundamentalmente 'una entrevista personal en la que el trabajador relata cada una de sus actividades, documentación que maneja, responsabilidades asignadas y sugerencias de mejora' [30] realizada in situ y por lo general dentro de los horarios de trabajo, es necesario contar con la aprobación de la alta dirección o del responsable del área de gestión pues eventualmente deberá detener sus actividades para atender a los requerimientos de información. Un aspecto a considerar en la planificación de vistas a los entrevistados es considerar el flujo de trabajo y de ser posible realizar la acción del levantamiento en las horas de menor ejecución de actividades para disponer del tiempo necesario y sobre todo la total atención requerida para asegurar una mayor calidad y veracidad de la información provista.

\subsection{Segunda etapa: Levantamiento de procesos (Etapa principal)}

Para que se de inicio al levantamiento de procesos es necesario que la alta dirección de la organización comunique a todos los colaboradores sobre el desarrollo de esta actividad, las motivaciones, ventajas que tendrá para la empresa, cronograma y la actitud adecuada hacia los responsables de llenar los instrumentos de trabajo.

Se debe hacer hincapié en la importancia de esta actividad para documentar adecuadamente los procesos, explicar los objetivos que tiene, solventar las dudas que se presentaran, la predisposición de colaboración con el responsable del levantamiento y la necesidad de informar con el mayor apego a la realidad las actividades que cada uno realiza. 


\subsubsection{Ingreso de la información}

Inicia cuando el responsable del levantamiento de la información se acerca al puesto de trabajo del colaborador que será objeto de la entrevista; posterior a la correspondiente presentación y explicación de la naturaleza de la actividad a realizar, debe hacer hincapié en la importancia de la veracidad de los datos que proporciona.

El instrumento utilizado para la recolección de datos se denomina hoja de caracterización, esta ficha de trabajo debe su nombre a que en ella se caracterizan o registran las particularidades del proceso, de modo que claramente se distingan [31] todas las actividades que se realizan, además de la identificación y descripción cuantitativa y cualitativa [32] de los elemento de entrada y salida, así como la documentación asociada, los recursos que se emplean y los productos del mismo y datos generales como el nombre del proceso, el responsable y objetivo.

El ingreso de información se debe realizar a medida que el colaborador la provea, de tal forma que se asegure no pasar por alto ningún detalle, una vez finalizado el ingreso de un proceso y previo a continuar con el siguiente es necesario hacer una revisión de la toda la información, seguido de la firma de conformidad que dará respaldo al registro que se acaba de generar. En la tabla que se presenta (Tabla 3) se puede observar la hoja de caracterización (anverso) y el reverso de la misma (Tabla 4). Es importante señalar que se debe usar una hoja distinta para cada proceso.

El lado opuesto permite el ingreso de información adicional al procesos como las condiciones en que se desarrolla como ambientales, de infraestructura, equipo entre otros, también permite poder registra sugerencias, opiniones del responsable del proceso, posibles acciones u oportunidades de mejora, ubicación, entre otras.

La actividad finaliza una vez que el responsable del ingreso de información registró todos los procesos y le informe al colaborador que se realizará una segunda visita para validar los datos. Entre las novedades que se puedan presentar al finalizar esta actividad es el aparecimiento de procesos que se están ejecutando y que podrían no estar documentados conforme la información de la Tabla 2 (información legal y/o estatutaria)

La siguiente imagen muestra una hoja de caracterización con información levantada 'in situ' y provista por un colaborador de la Dirección de Desarrollo Académico como parte de la elaboración de la estructura documental para la aplicación del enfoque de procesos en la dirección. 
Table 3

Hoja de caracterización (anverso).

\begin{tabular}{|c|c|c|c|c|c|c|}
\hline \multirow{5}{*}{$\begin{array}{l}\text { Número } \\
\text { de } \\
\text { proceso: }\end{array}$} & \multicolumn{5}{|c|}{$\begin{array}{l}\text { LEVANTAMIENTO DE PROCESOS } \\
\text { (HOJA DE CARACTERIZACIÓN) }\end{array}$} & \multirow[t]{5}{*}{ Hoja: } \\
\hline & \multicolumn{5}{|c|}{$\begin{array}{l}\text { Área de gestión } \\
\text { Dependencia }\end{array}$} & \\
\hline & \multicolumn{2}{|c|}{ Nombre del proceso } & & Código & & \\
\hline & \multicolumn{2}{|c|}{ Responsable } & & Autor & & \\
\hline & \multicolumn{2}{|l|}{ Objetivo } & & Fecha & & \\
\hline \multicolumn{3}{|l|}{ Entradas } & \multirow[t]{2}{*}{ ACTIVIDADES } & \multicolumn{3}{|l|}{ Salidas } \\
\hline $\begin{array}{l}\text { Fecha } \\
\text { de Ref. }\end{array}$ & $\begin{array}{l}\text { Proviene } \\
\text { de: }\end{array}$ & Tipo: & & $\begin{array}{l}\text { Se dirije } \\
\text { a: }\end{array}$ & $\begin{array}{l}\text { Proviene } \\
\text { de: }\end{array}$ & $\begin{array}{l}\text { Fecha } \\
\text { de Ref. }\end{array}$ \\
\hline & & & & & & \\
\hline & & & & & & \\
\hline & & & & & & \\
\hline & & & & & & \\
\hline & & & & & & \\
\hline & & & & & & \\
\hline & & & & & & \\
\hline & & & & & & \\
\hline & & & & & & \\
\hline & & & & & & \\
\hline & & & & & & \\
\hline & & & & & & \\
\hline & & & & & & \\
\hline & & & & & & \\
\hline & & & & & & \\
\hline
\end{tabular}

\subsubsection{Tratamiento de la información}

Con los datos obtenidos es necesario ingresar en un formato que permita ordenar adecuadamente según la naturaleza de la información y empezar a elaborar los documentos de gestión. Aunque esto se lo puede hacer de diversas maneras lo importante es que se puedan diferenciar claramente los elementos del proceso. El primer elemento a diferenciar son las características de identificación, es decir el nombre, objetivo, responsable del proceso. También se pueden ingresar la información respecto de los elementos de entrada, necesidades de recurso, salidas (productos) y el indicador que permitirá su gestión y control [33] entre otros; a esta parte de la documentación se le denomina ficha de proceso, a continuación un ejemplo de su estructura.

A continuación, se debe documentar el desarrollo de las actividades, es decir el procedimiento del proceso, existe dos formas principales en la que se puede realizar, sin embargo la decisión será resultado de la identificación de la cultura organizacional de los trabajadores de la empresa, entendida como 'el patrón general de conductas, 
Table 4

Hoja de caracterización (reverso).

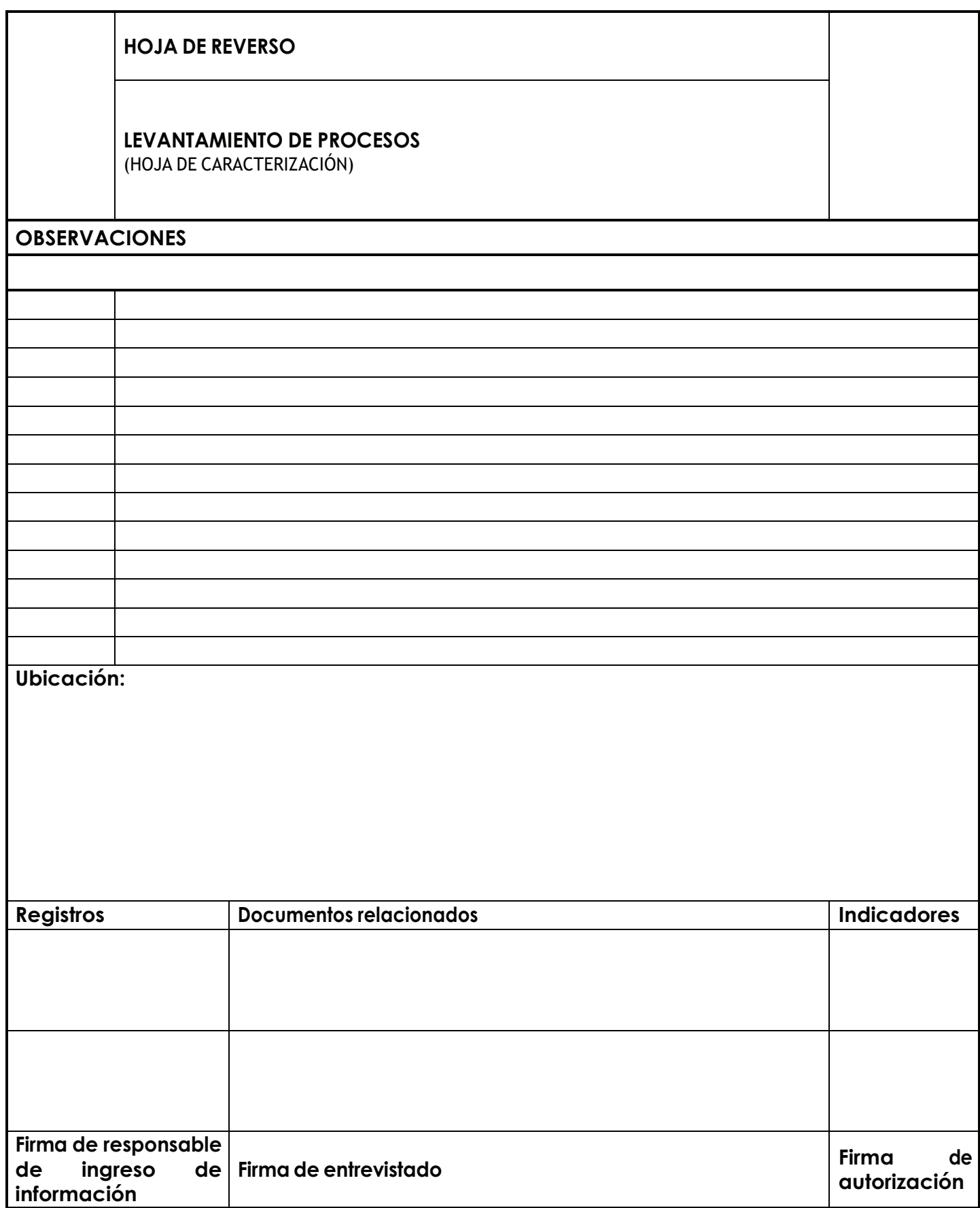

creencias y valores compartidos por los miembros de una organización' [34] o dicho de otra manera el lenguaje en que todos se comunican entre sí.

La primera forma en que se puede documentar las actividades es mediante un listado secuencial de ellas (Tabla 6), la segunda forma es mediante el uso de gráficos (Tabla 7) Ilamado como el diagrama de flujo. 

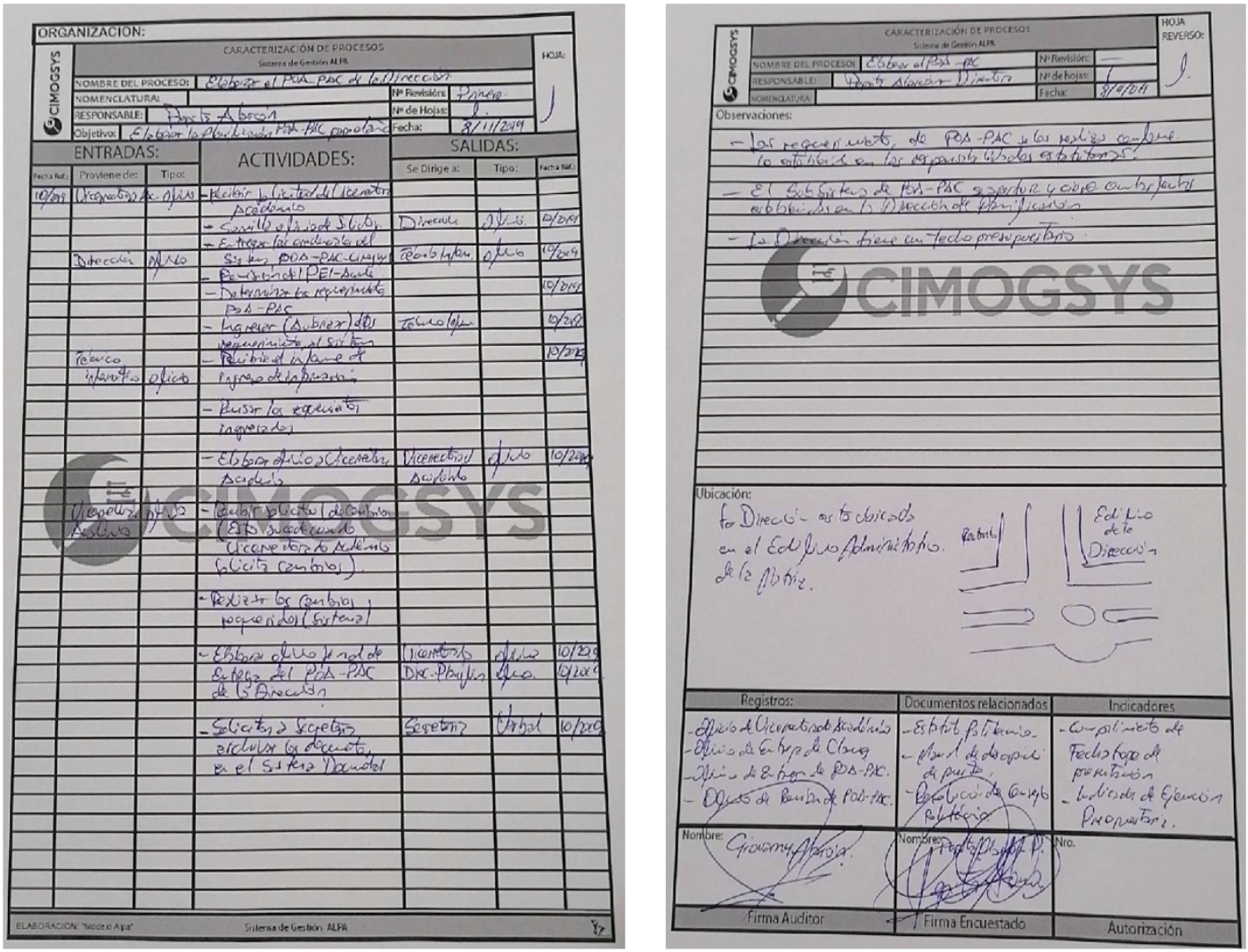

Figure 1

Hojas de caracterización empleadas en la Dirección de Desarrollo Académico (digitalizadas).

Table 5

Ficha de proceso.

\begin{tabular}{|c|c|c|c|c|}
\hline \multicolumn{5}{|c|}{ FICHA DE PROCESO } \\
\hline \multicolumn{5}{|l|}{ Nombre } \\
\hline \multicolumn{5}{|l|}{ Objetivo } \\
\hline \multicolumn{5}{|l|}{ Responsable } \\
\hline \multicolumn{5}{|c|}{ Lugar de Ejecución } \\
\hline \multicolumn{5}{|l|}{ Ingresos } \\
\hline \multicolumn{5}{|l|}{ Entradas } \\
\hline Recursos & Humanos & $\begin{array}{l}\text { Infraestructura } \\
\text { física }\end{array}$ & $\begin{array}{l}\text { Infraestructura } \\
\text { tecnológica }\end{array}$ & $\begin{array}{l}\text { Equipo - } \\
\text { herramientas }\end{array}$ \\
\hline \multicolumn{5}{|c|}{ Documentación } \\
\hline \multicolumn{5}{|l|}{ Salidas } \\
\hline \multicolumn{5}{|l|}{ Productos } \\
\hline \multicolumn{5}{|l|}{ Sub productos } \\
\hline \multicolumn{5}{|c|}{ Información (documentos) } \\
\hline \multicolumn{5}{|c|}{ Elementos de gestión } \\
\hline \multicolumn{5}{|l|}{ Indicador } \\
\hline Cualitativo & Valores & & & \\
\hline Cuantitativo & Fórmula & & & \\
\hline
\end{tabular}


Table 6

Procedimiento (listado de actividades).

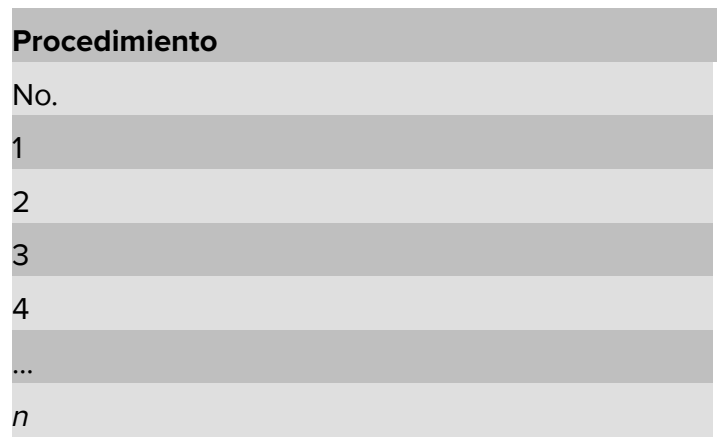

Actividades

Table 7

Procedimiento (diagrama de flujo).

\section{Procedimiento}

No.

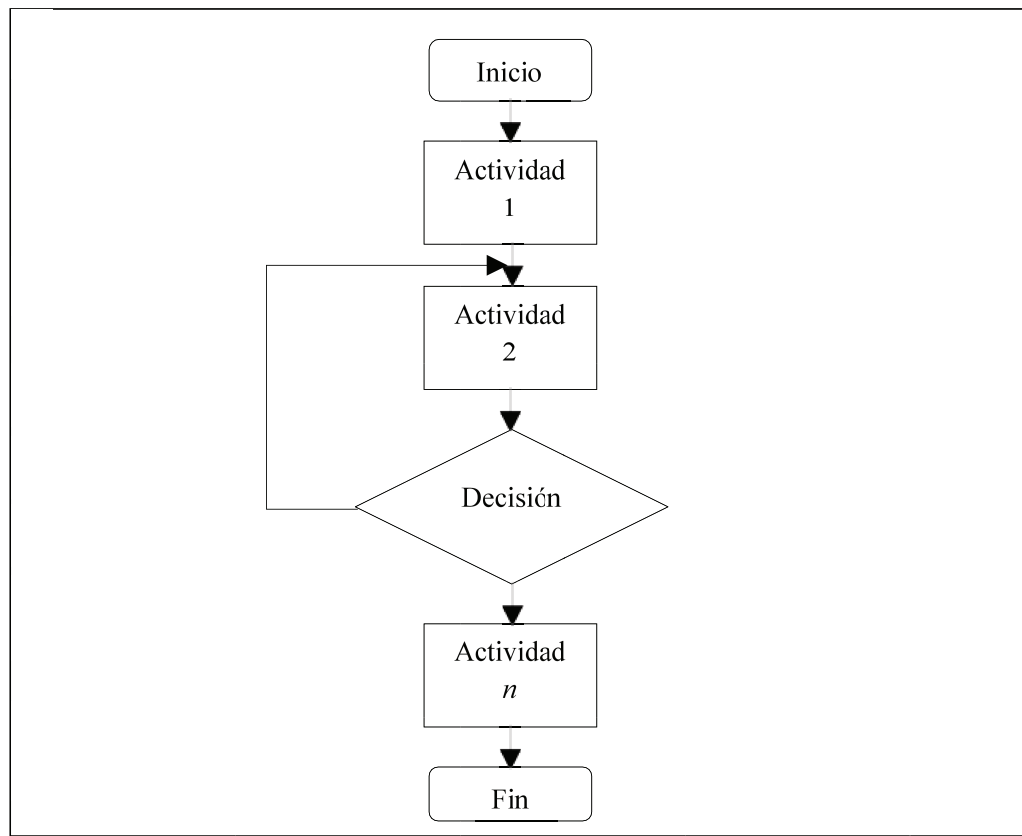

Una de las ventajas del listado de actividades es su fácil interpretación, una lectura y revisión del texto será suficiente para conocer el desarrollo del proceso, su secuencia y resultados previstos. Por otro lado, a pesar que el uso de diagramas de flujo requiere el conocimiento previo de la simbología empleada y su significado, tiene entre sus principales ventajas la identificación visual de la secuencia de actividades y como interactúan entre sí, no solo con el responsable del proceso sino también con otros colaboradores, se tiene una visión global del proceso; sin embrago y como se manifestó en líneas anteriores dependerá de la cultura organizacional y el lenguaje que maneja 
la empresa, lo que no significa que con actividades de capacitación se pueda asegurar que todos los colaboradores puedan adquirir el conocimiento necesario para el uso de los símbolos.

\subsubsection{Validación de la información}

Cuando se han documentado los procesos, es necesario realizar una segunda visita al colaborador para revisar y corroborar la información, esta actividad permitirá identificar posibles inconsistencia o falta de lógica en la secuencia de las actividades; además se podrán dotar de mayor detalle si el caso lo amerita.

\subsection{Tercera etapa: Documentación de los procesos}

La parte final es la elaboración del o los manuales que contendrán los procesos documentados, por lo general este documento se denomina como manual de procesos, que tiene como propósito orientar las acciones que deben guiar a todos los colaboradores [35].

Este manual una vez concluido deberá ser entregado a la alta dirección para que sea revisado y de ser el caso modificado para que se ajuste a la realidad de la empresa; una vez finalizado se debe aprobar con la generación de una evidencia como un acta de aprobación, de esta manera queda legalizado y de uso obligatorio y con los procesos que contiene estandarizados.

La emisión del manual de procesos dependerá de la organización y de su sistema de gestión documental [36]; se deben tener en consideración que se pueden usar diferentes tipos de medios [37] como el material impreso o como sucede en la actualidad (en el que se busca la reducción del uso de hojas impresas) el formato digital.

Después de su emisión la organización debe asegurarse de comunicar a todos los colaboradores de la existencia del manual, capacitar sobre su uso adecuado y distribuirlos en los puntos de uso para que cumpla su propósito de ser fuente de consulta entre otros mencionados con anterioridad.

Es importante indicar que el manual y sus procesos se deben versionar, es decir indicar que versión es con el objetivo de poder controlar el uso vigente del documento, y cuando sea actualizado y emitido una nueva edición, informar a los usuarios la obsolescencia del manual anterior y la vigencia de la nueva edición.

En este punto una adecuada práctica administrativa es llevar un registro de los documentos entregados, de esta forma se podrá retirar la versión obsoleta y entregar la nueva; en el caso del uso de medio digitales asegurar procesos de comunicación efectiva para dar a conocer que el manual entregado con anterioridad ha sido descontinuado. 
La siguiente combinación de imágenes (Figura 2) muestra la portada del manual de procesos de la Escuela de Administración de Empresas de la Facultad de Administración de Empresas (FADE), correspondiente a la segunda edición, y como se puede observar en la segunda imagen la tabla de registro de ediciones en la que se pueden identificar las fechas de cada versión, el motivo de la edición y la fecha de expedición entre los principales datos.
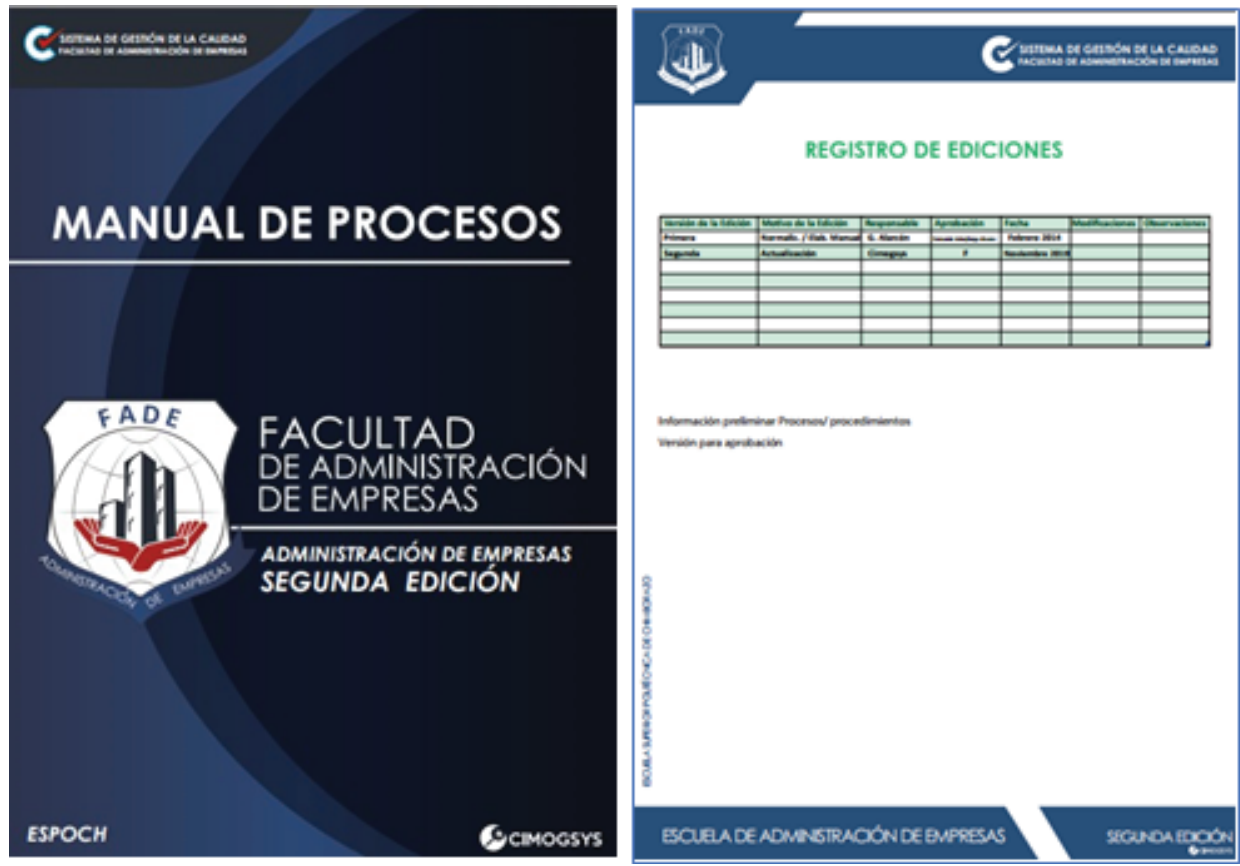

Figure 2

Portada del manual de procesos de la Escuela de Administración de Empresas (FADE) segunda edición, y hoja con la tabla de registro de ediciones.

Uno de los beneficios de una documentación de procesos bien realizada es la utilidad que tiene, pues además de evidenciar el modelo de gestión que emplea la empresa para actividades de control y verificación ya sea por unidades internas u organismos externos en la verificación del cumplimiento de los requisitos legales y estatutarios aplicables, se utilizan también como:

- Fuente de consulta para la ejecución de procesos,

- Instrumento para capacitación al personal (colaboradores),

- Instrumento de inducción a nuevo personal,

- Identificación de procesos y actividades duplicadas,

- Identificación de cuellos de botella,

- Mejoramiento de procesos,

- Insumo en la planificación de auditorias de gestión (de procesos), 
- Entre otros.

Debido a la naturaleza cambiante tanto del entorno de la organización, como de los requerimientos de clientes y emisión de nuevas normativas por parte de los organismos de control, la actualización orgánica y de su modelo de gestión será una actividad que requerirá periodicidad, de forma tal que la aplicación de la metodología aquí expuesta no será de única aplicación, sino que dependiendo de las necesidades se la realizara en distintas ocasiones. Dependiendo del giro del negocio o las actividades a las que se dedica la empresa, es adecuado que se planifique el levantamiento de procesos con fines de actualización de manera planificada y recurrente para asegurar que se cuente con información actualizada. Cabe indicar que una vez aprobados los procesos documentados, aquellos procesos que no formaban parte de estatutos y reglamentos conforme la información de la Tabla 2, toman carácter legal y a partir de ese momento deben ser gestionados, controlados y mejorados al igual que el resto de procesos. También es origen y genera la necesidad de una actualización de los mencionados documentos legales.

\section{Resultados y Discusión}

La metodología propuesta en el presente trabajo ha sido desarrollada y evolucionada a lo largo de ocho años como parte de las actividades utilizadas dentro de distintos proyectos de investigación para la aplicación del enfoque de procesos en varias unidades de gestión en la Escuela Superior Politécnica de Chimborazo (ESPOCH) obteniendo resultados positivos.

Entre los principales efectos obtenidos son la reducción del tiempo de la actividad de levantamiento de procesos, pues al estar estandarizada y debidamente socializada su aplicación es mas eficiente; también se pudo desarrollar la actividad en repetidas ocasiones a lo largo de los años en que se realizaron aplicaciones del enfoque de procesos y con distintos responsables de levantamiento de información obteniendo siempre los mismo resultados. El mantenimiento de la metodología también ha generado confianza de parte de los colaboradores en la institución pues ya conocen su metodología y su apertura a brindar información ha sido mayor; extraídos del manual de procesos de la Escuela de Administración de Empresas (EAE) de la Facultad de Administración de Empresas (FADE), las imágenes que se presentan corresponden a la documentación de un proceso en particular en el manual edición primera del año 2014 (Figura 3) y la siguiente (Figura 4) a la documentación de un proceso especifico en el manual edición segunda del año 2019 (manual que forma parte del sistema de gestión de la calidad certificado), para este ultimo manual se aplico la metodología en con el 
objeto de fortalecer la gestión de la facultad y contribuir a la conformidad exigida por la norma de calidad en proceso de implementación.
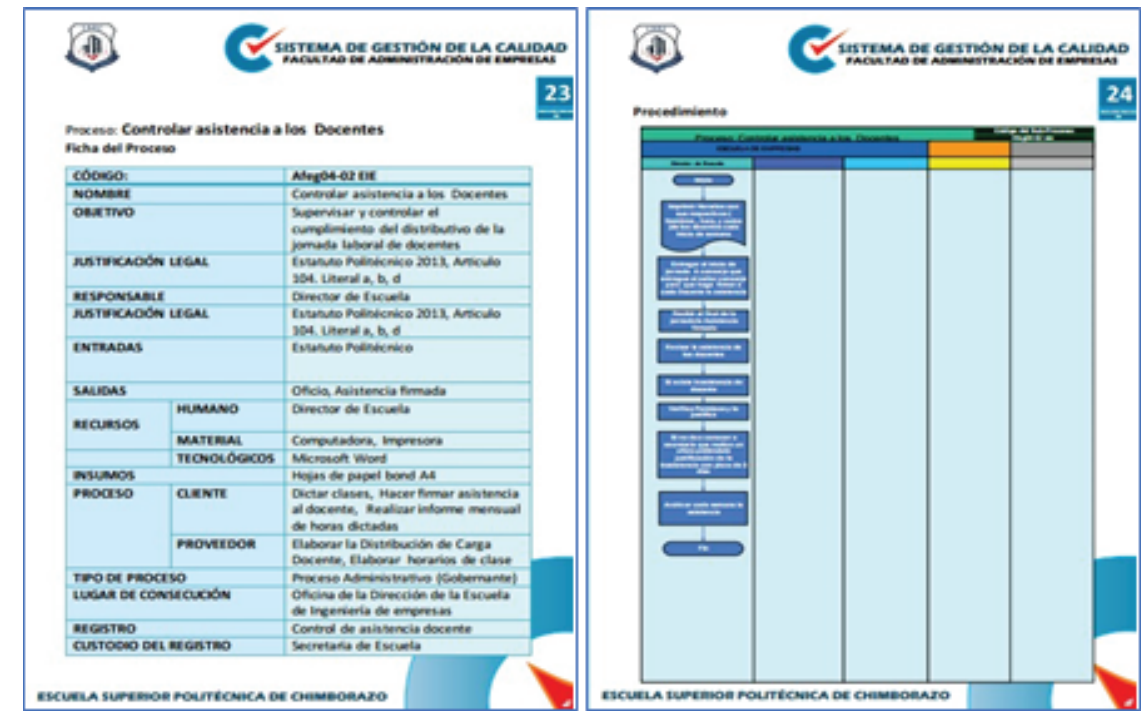

Figure 3

Ejemplo de procedimiento documentado (ficha de proceso y procedimiento) del Manual de Procesos de la Escuela de Administración de Empresas (FADE) primera edición año 2014.

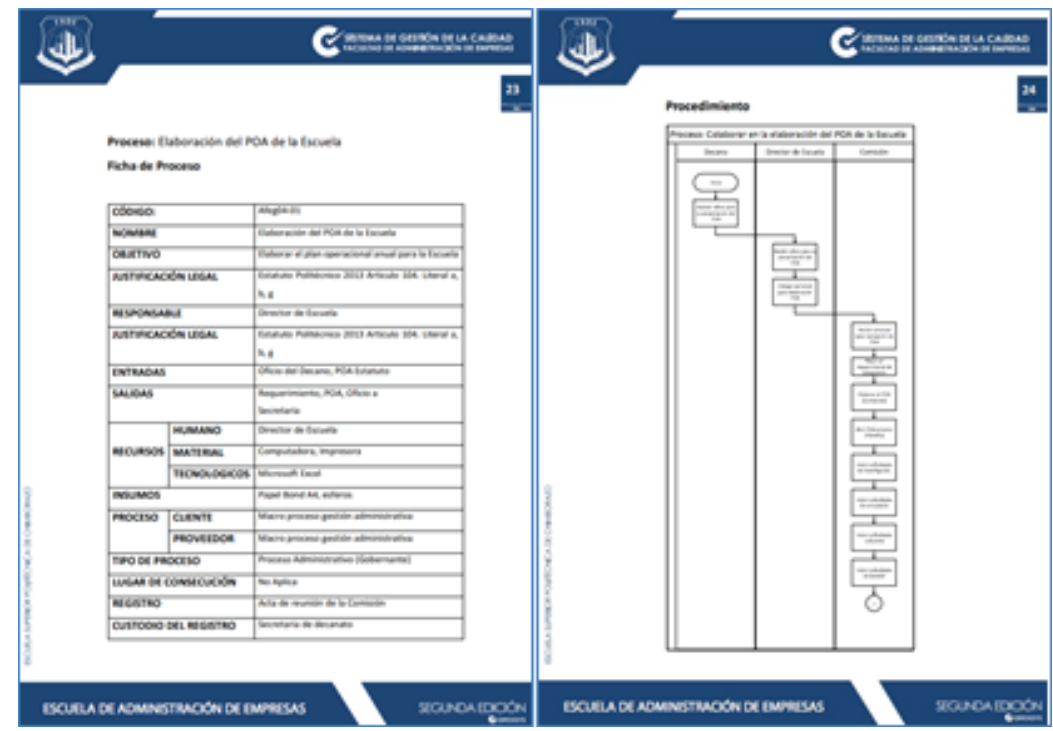

Figure 4

Ejemplo de procedimiento documentado (ficha de proceso y procedimiento) del Manual de Procesos de la Escuela de Administración de Empresas (FADE) segunda edición año 2019.

Aplicando la misma metodología se documentaron los procesos de la Dirección Financiera de la Escuela Superior Politécnica de Chimborazo, este caso se trabajo con un estudiante incorporado al proyecto de investigación quién después de la capacitación en el manejo de la metodología y uso de los instrumentos, formatos y estándar 
de manual de procesos logró identificar todos los procesos. La Figura 5 muestra un proceso documentado.
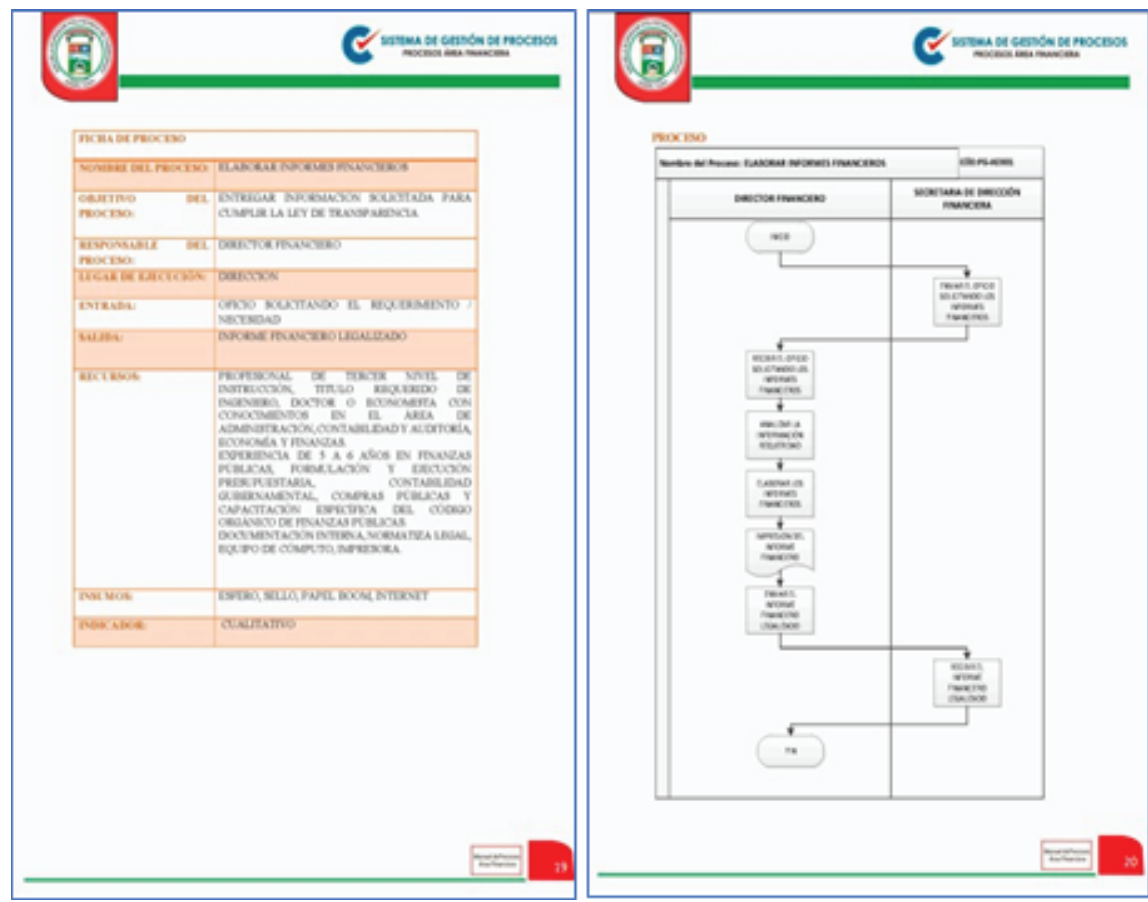

Figure 5

Ejemplo de procedimiento documentado (ficha de proceso y procedimiento) del Manual de Procesos de la Dirección Financiera (ESPOCH) primera edición año 2016.

Entre los principales resultados obtenidos fue el apoyo en la elaboración de la documentación de un sistema de gestión de la calidad (SGC) conforme la norma internacional ISO 9001:2015 de la Facultad de Administración de Empresas de la Escuela Superior Politécnica de Chimborazo, unidad académica que tomó la decisión estratégica de elaborar e implementar el SGC con el objetivo de poder mejorar la eficiencia de sus procesos y elevar los niveles de satisfacción de sus partes interesadas. Este trabajo se llevó en conjunto con la Comisión del Sistema de Gestión de la Calidad SGC Fade y su equipo de auditoría interna.

La auditoría de conformidad por parte de los auditores de la empresa certificadora tuvo entre sus principales actividades la verificación de los procedimientos documentados, aspecto fundamental para llegar a obtener la certificación internacional, y que al demostrar adecuadamente la identificación y documentación de los procesos [38] y procedimientos, se obtuvo como resultado de la auditoría el 'consejo' a la empresa certificadora la expedición del certificado de conformidad y de cumplimiento de la citada norma, hito de trascendental importancia debido a que es la primera facultad de la institución en conseguir este logro y una de las pocas facultades de administración del país en contar con la certificación de conformidad de su sistema de gestión de la calidad conforme la ISO 9001:2015, la Figura 6 muestra la publicación en la red social 
oficial de la facultad del día 31 de enero de 2020 dando a conocer el resultado del informe de la auditoría principal de certificación.

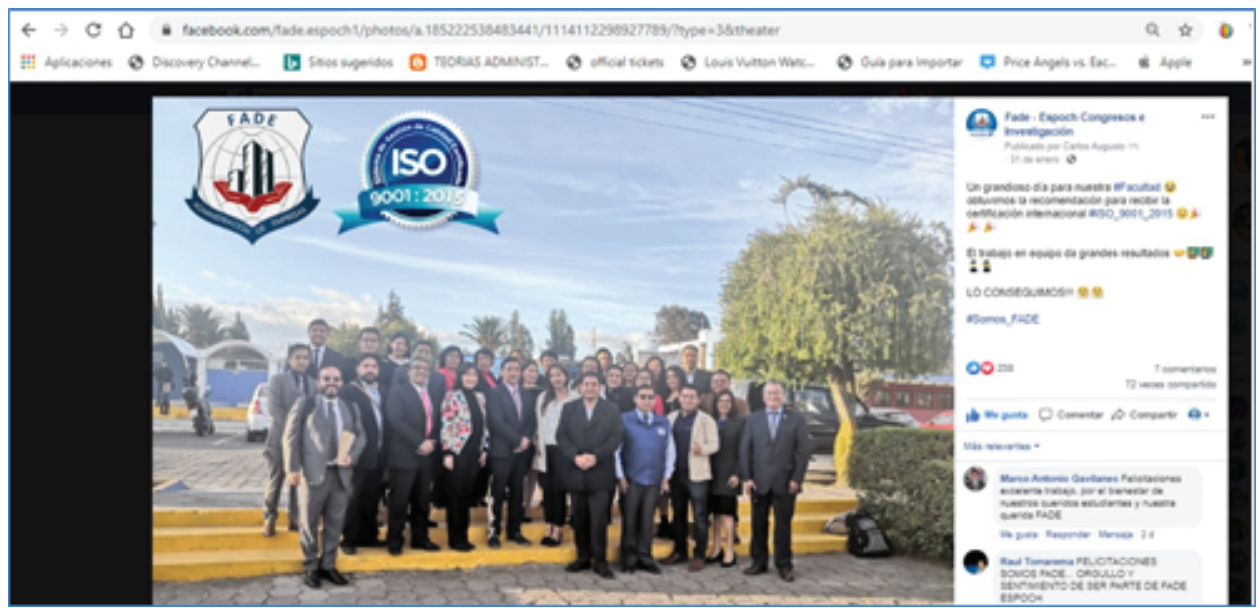

Figure 6

Publicación en el medio oficial (Facebook) del resultado de la auditoria principal del sistema de gestión de la calidad Fade, informando la recomendación de la entrega de la certificación a la facultad.

\section{Conclusiones}

El levantamiento de procesos es una actividad recurrente que se realiza con frecuencia en todo tipo de organizaciones, mas aún desde el reconocimiento que las normas de calidad han obtenido a nivel internacional, normas que entre sus puntos mas relevantes recomiendan la aplicación del enfoque de procesos como sucede en el punto 1,3 de la ISO 9001:2015, en la que se menciona de manera explícita que una organización que pretenda aumentar la satisfacción del cliente debería aplicar el modelo de gestión por procesos. En nuestro país esta tendencia tomó el carácter de legal y obligatoria con la expedición de las normas técnicas que rigen su uso en las instituciones públicas; por su parte las organizaciones privadas están orientado su modelo de gestión siguiendo estos preceptos motivadas por sus resultados.

Dada esta tendencia, las administraciones de las empresas y organizaciones casi de manera general realizan el encargo a uno o varios de sus trabajadores para levantar la información de los procesos y documentarlos, en muchos de los casos sin antecedentes o experiencia previa. No considerado como relevante, la acción de documentar los procesos no ha sido objeto de un estudio que permita estandarizar sus actividades de tal forma que su realización sea efectiva y menos aún se ha procurado el establecimiento de los instrumentos de trabajo. La metodología propuesta resultó ser efectiva en todos los casos aplicados y permitió no solo documentar los procesos y dar inicio a la elaboración de los correspondientes manuales de gestión, sino que también redujo 
el tiempo de ejecución de esta actividad, guió la labor de diferentes colaboradores encargados incluso sin conocimientos previos y aseguró su cumplimiento a pesar del cambio de responsables en algunos casos logrando su objetivo.

Una implicación de este trabajo es la posibilidad de incorporar el estándar propuesto a la metodología que una organización pudiera establecer para planificar, diseñar y elaborar un sistema de gestión de procesos, o sistema de gestión de calidad, su mantenimiento y reajuste respectivo con la aplicación frecuente y planificada para la actualización de procesos. También se puede abordar la temática de elaboración de la estructura documental como fundamento de un sistema de gestión de calidad y la planificación de auditorías de procesos.

\section{References}

[1] Ambrosone M. La administración del riesgo empresarial: Una responsabilidad de todos-el enfoque coso. Amazon news. 2007. Disponible en: https: //s3.amazonaws.com/academia.edu.documents/38727089/COSO.pdf?response-c ontent-disposition=inline\{\%\}3B\{\%\}20filename\{\%\}3DCOSO_ERM.pdf\&X-Amz-Algorit hm=AWS4-HMAC-SHA256\&X-Amz-Credential=AKIAIWOWYYGZ2Y53UL3A\{\%]2F 20200130\{\%\}2Fus-east-1\{\%\}2Fs3\{\%\}2Faws4_request\&X-Amz-Date=20200130T 131852Z\&X-Amz-Expires $=3600 \& X-A m z-S i g n e d H e a d e r s=h o s t \& X-A m z-S i g n a t u r e$

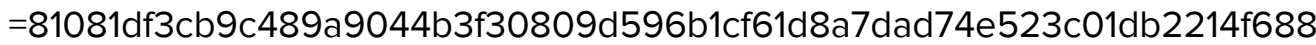

[2] Viloria N. Factores que inciden en el sistema de control interno de una organización. Actualidad Contable Faces. 2005;8:87-92.

[3] De Souza EM, Aguiar CA. Publicações póstumas de Henri Fayol: revisitando sua teoria administrativa. RAM. Revista de Administração Mackenzie. 2011;1: 204-227.

[4] Ramos, OP. Taylor y fayol: Vigencia de los principios clásicos. Revista Adelante Ahead. 2010;1:67-76.

[5] Quiroga, LA. Gestión de información, gestión del conocimiento y gestión de la calidad en las organizaciones. ACIMED. 2002;5:7-8.

[6] Bustelo Ruesta C, Iglesias AR. Gestión del conocimiento y gestión de la información. Boletín del Instituto de Andaluz de Patrimonio Histórico. 2001;34:226-230.

[7] Dextre Flores JC, Del Pozo R. ¿Control de gestión o gestión de control?. Contabilidad y Negocios. 2012;14:69-80.

[8] Freire, FC. Los nuevos modelos de gestión de las empresas mediáticas. Estudios sobre el Mensaje Periodístico. 2010;16:13-30.

[9] Palma H. La gestión empresarial, un enfoque del siglo XX, desde las teorías administrativas científica, funcional, burocrática y de relaciónes humanas. Escenarios. 2011;1:38-51. 
[10] Chica MA, Villada AV, Restrepo EA. Análisis de cargas laborales y actualización de los manuales de funciones y requerimientos Hospital San José de Marsella. Pereira; 2013.

[11] González, IR, González A, Viamontes PN, Sotolongo, PS. Metodología de Diseño Organizacional integrando enfoque a procesos y competencias. Ingeniería Industrial. 2012;2:188-199.

[12] Parra, GJA. Propuesta de gestión organizacional por procesos para la facultad de administración de empresas de la ESPOCH [Tesis de posgrado]. Guayaquil: FUNIBER; 2014.

[13] Álvarez BM. Importancia de la implementación del manual de procesos y procedimientos para el logro de los objetivos de las escuelas populares del deporte pertenecientes al instituto de deportes y recreación de Medellín. Medellín: Colombia; 2007.

[14] ISO. 2015. www.iso.org. ISO 9000:2015(es) Sistemas de gestión de la calidad — Fundamentos y vocabulario. Ginebra: Organización Internacional de Estandarización.

[15] ISO. 2005. ISO 9000:2005(es) Sistemas de gestión de la calidad - Fundamentos y vocabulario. Ginebra: Organización Internacional de Estandarización.

[16] Escalante P, Hulett N. Importancia de la auditoría de estados financieros para las Pyme’s: Una revisión documental. Actualidad Contable Faces. 2010;20:19-28.

[17] Yánez J, Yánez R. Auditorías, mejora continua y normas ISO: Factores clave para la evolución de las organizaciones. Ingeniería Industrial. Actualidad y Nuevas Tendencias. 2012;9:83-92.

[18] Sarmiento NLI, Ramírez VM, Berdugo C. Aplicación de una Metodología de Mejora de Procesos basada en el Enfoque de Gestión por Procesos, en los Modelos de Excelencia y el QFD en una empresa del sector de confecciones de Barranquilla. Ingeniería y Desarrollo. 2004;16:45-58.

[19] ITU. BS.1387: Método para la medición objetiva de la calidad de audio percibida (Rec. UIT-R BS.1387 ed.). (I. C. world, Ed.) Ginebra: ITU: Committed to connecting the world; 1998.

[20] Zaratiegui J. La gestión por procesos: Su papel e importancia. Economía industrial. 1999;300:81-82

[21] Becerra AA, Fernández ME, Robaina AD. Dirección por procesos en la Universidad. Ingeniería Industrial. 2013;1:87-95.

[22] Fernández Z. La estructura organizativa: Un análisis contingente. Investigaciones Económicas (segunda época). 1986;3:467-82.

[23] Rotundo ZG, Martínez MA, Hernández A. Modelo teórico conceptual de la estructura organizativa: Un análisis contingente. Ciencia y sociedad. 2009;4:618-640.

[24] Parra AGJ, Parra PI, Arias GSE. La elaboración del mapa de procesos para una universidad ecuatoriana. Revista Espacios; 2019;40. 
[25] Ortega PE, Guilló TJ, Moliner PJ, Azorín MJ, Gamero LMD. Certificación en calidad, resultados empresariales y estructura organizativa en el sector hotelero español. Intangible Capital. 2013;1:199-224.

[26] Bravo DL, García TU, Hernández M, Ruiz VM. La entrevista, recurso flexible y dinámico. Investigación en educación médica 2.7. 2013;7:162-167.

[27] Lugo AH. Gestión por procesos. 2003;1:3-6.

[28] Casal J, Mateu E. Tipos de muestreo. Epidem. Med. Prev. 2003;1:3-7.

[29] Otzen T, Manterola C. Técnicas de muestreo sobre una población a estudio. International Journal of Morphology. 2017;35(1),227-232.

[30] Parra GJA, Gualán LDA, Parra PIA, Chávez GMA. Aplicación del enfoque de procesos en el área financiera de la escuela superior politécnica de chimborazo. Revista Caribeña de Ciencias Sociales. 2017.

[31] Real Academia Española. 2020. Obtenido de https://dle.rae.es/caracterizar

[32] FAO. La situación de los recursos zoogenéticos mundiales para la alimentación y la agricultura. Roma: Barbara Rischkowsky y Dafydd Pilling; 2010.

[33] Santos LM, Dante PG, Ponjuán TD. Panorámica sobre la medición del conocimiento organizacional. Acimed. 2009;6.

[34] Estrada JGS, Pupo, JCG, Rodríguez YBM, Andalia RC. Clima y cultura organizacional: dos componentes esenciales en la productividad laboral. Acimed. 2009;4:67-75.

[35] Vergara M. Diseño del manual de procesos y procedimientos del área Recursos Humanos del ISS, por tipo de seccional de acuerdo a complejidad y tamaño (modelo seccional Cundinamarca y DC). Cundinamarca; 2007.

[36] Dante PG. Gestión documental, gestión de información y gestión del conocimiento: Evolución y sinergias. Comunicación preliminar. Ciencias de la Información; 2015.

[37] ISO. 2008. Sistemas de gestión de la calidad - Requisitos. 42. Ginebra: Organización Internacional de Estandarización.

[38] Fonseca J, Muñóz N, Cleves J. El sistema de gestión de calidad: Elemento para la competitividad y la sostenibilidad de la producción agropecuaria colombiana. Revista de Investigación Agraria y Ambiental. 2011:1;9-22. 